01

\title{
Квазипериодические бифуркации четырехчастотных торов в кольце пяти связанных осцилляторов ван дер Поля с различными видами диссипативной связи
}

\author{
(C) Н.В. Станкевич, ${ }^{1}$ А.П. Кузнецов, ${ }^{2}$ Е.П. Селезнев ${ }^{2}$ \\ ${ }^{1}$ Саратовский государственный технический университет им. Ю.А. Гагарина, \\ 410054 Саратов, Россия \\ ${ }^{2}$ Саратовский филиал Института радиотехники и электроники им. В.А. Котельникова, \\ 410019 Саратов, Россия \\ e-mail: stankevichnv@mail.ru
}

(Поступило в Редакцию 16 октября 2016 г.)

Представлено численное исследование пяти связанных в кольцо осцилляторов ван дер Поля. Рассмотрены случаи различных типов связи: диссипативная, активная, а также диссипативная и активная с единичной инверсией знака связи. Изучены бифуркации, наблюдаемые при переходе от пятичастотного тора к четырехчастотному тору. Обнаружена возможность квазипериодической бифуркации Хопфа в такой системе.

DOI: $10.21883 /$ JTF.2017.06.44525.2063

Динамика связанных осцилляторов представляет достаточно большой интерес в различных областях науки. За последнее время проведено достаточно большое количество исследований в этом направлении [1-4]. Одним из важных и интенсивно обсуждаемым аспектом динамики связанных осцилляторов является исследование многочастотных квазипериодических колебаний в такого рода системах [1-6]. К настоящему времени эволюция двумерного тора изучена досконально, чего не скажешь об исследованиях систем, в которых аттракторами являются торы более высокой размерности. В связи с этим представляется интересным исследование систем, в которых наблюдаются многомерные торы и их различные трансформации.

Одним из объектов, в котором можно наблюдать многомерные торы, например, размерности пять, является система пяти связанных автогенераторов. На рис. 1 приведена блок-схема для случая пяти автогенераторов, связанных в кольцо. В случае слабой связи синхронизация между автогенераторами отсутствует, аттрактором

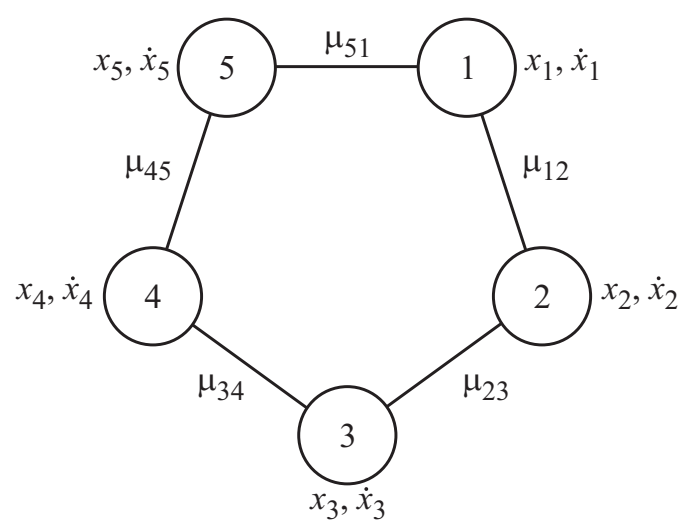

Рис. 1. Блок-схема пяти осцилляторов, связанных в кольцо, являющихся объектом исследования. данной системы будет пятимерный тор. При увеличении связи будет происходить подстройка и захват частот генераторов и будет наблюдаться переход от пятичастотного тора (5-тор) к четырехчастотному тору (4-тор) и т. д. Данные переходы ассоциируются с двумя основными бифуркациями квазипериодических режимов. Одна из них - это квазипериодическая седло-узловая бифуркация, или седло-узловая бифуркация торов. В этом случае рождение тора более высокой размерности происходит в результате слияния устойчивого и седлового торов, имевших на единицу меньшую размерность [7]. Именно такие бифуркации, сопровождающиеся жестким изменением колебательного режима, характерны для случая резонансных взаимодействий. Однако для реализации сценария Ландау-Хопфа принципиальным является наличие квазипериодических бифуркаций Хопфа: мягкое рождение тора более высокой размерности. К факторам, влияющим на тип бифуркации, реализующейся в системе, относят топологию связи, распределение собственных частот элементов ансамбля, уровень диссипации в системе [2]. В настоящей работе рассматривается динамика пяти связанных в кольцо осцилляторов ван дер Поля с диссипативной, активной и специальной связями с инверсным знаком. В зависимости от типа связи будут исследованы условия возникновения квазипериодической бифуркации Хопфа.

Объектом исследования является модель, описывающая динамику пяти связанных в кольцо осцилляторов ван дер Поля. Математические уравнения модели запишем следующим образом:

$$
\begin{aligned}
& \ddot{x}_{i}-\left(\lambda-x_{i}\right)^{2} \dot{x}_{i}+\omega_{i} x_{i}+\mu_{i, i-1}\left(\dot{x}_{i}-\dot{x}_{i-1}\right) \\
& \quad+\mu_{i, i+1}\left(\dot{x}_{i}-\dot{x}_{i+1}\right)=0 .
\end{aligned}
$$

Здесь $x_{i}, \dot{x}_{i}$ - динамические переменные, характеризующие поведение $i$-го осциллятора; $i$ - номер осциллятоpa, $i=(1-5) ; \lambda$ - параметр, ответственный за возбуж- 
дение автоколебаний $(\lambda=0$ соответствует бифуркации Андронова-Хопфа), для всех осцилляторов параметр выбирался одинаковым, $\lambda=0.5 ; \omega_{i}=\left(1+\frac{(i-1) \Delta}{4}\right)-$ собственная частота каждого осциллятора, $\Delta-$ частотная расстройка между осцилляторами, для всех численных экспериментов была зафиксирована равной единице $(\Delta=1) ; \mu_{i, j}$ - коэффициенты связи, задание которых будет обсуждаться далее. Связь носит диссипативный характер, т.е. задается через производные переменных соседних осцилляторов. Граничные условия, обеспечивающие кольцевую топологию связи, запишем следующим образом:

$$
\mu_{01}=\mu_{56}=\mu_{51}, \quad \dot{x}_{0}=\dot{x}_{5}, \quad \dot{x}_{6}=\dot{x}_{1} .
$$

На рис. 1 представлена схема нашей системы, организованная в виде кольца, связь между соседними осцилляторами определяется коэффициентами $\mu_{i, j}$.

Основная методология исследования многочастотных квазипериодических колебаний основана на анализе полного спектра показателей Ляпунова. Присутствие в сигнатуре дополнительных нулевых экспонент свидетельствует о наличии в системе дополнительных иррациональных частот. Одним из удобных методов исследования динамики таких систем является метод карт показателей Ляпунова [1], суть которого заключается в следующем: выбирается некоторая плоскость управляющих параметров, которая сканируется с небольшим шагом. Для каждой точки плоскости рассчитывается полный спектр показателей Ляпунова с помощью алгоритма Бенеттина, и в зависимости от значений показателей точка на плоскости параметров окрашивается в тот или иной цвет. Данный подход широко используется при анализе многочастотной квазипериодической динамики в низкоразмерных ансамблях осцилляторов $[1,2]$. В работе [7] была показана возможность различить квазипериодическую бифуркацию Хопфа от седло-узловой квазипериодической бифуркации по динамике показателей Ляпунова в зависимости от параметра в момент бифуркации. Идентификатором квазипериодической бифуркации Хопфа является равенство по абсолютному значению двух показателей Ляпунова, последующих за нулевыми. В случае квазипериодической седло-узловой бифуркации эти показатели отрицательные и не равны друг другу.

Исследование динамики системы в настоящей работе будет проводиться с помощью модифицированных карт показателей Ляпунова, на которых отдельным цветом будут обозначены области с отрицательными равными по абсолютной величине показателями Ляпунова. Такая модификация позволит диагностировать и различить линии квазипериодической бифуркации Хопфа и седлоузловой. В таблице представлено соответствие сигнатуры спектра показателей Ляпунова, реализующегося режима и его цветовое обозначение на картах показателей Ляпунова.
Итак, рассмотрим систему (1) с диссипативной и активной связями. Для наглядности исследуемой системы введем матрицу связи, описывающую топологию связи:

$$
\mu_{i j}^{1}=\left(\begin{array}{ccccc}
\mu_{51}+\mu_{12} & -\mu_{12} & 0 & 0 & -\mu_{51} \\
-\mu_{12} & \mu_{12}+\mu_{23} & -\mu_{23} & 0 & 0 \\
0 & -\mu_{23} & \mu_{23}+\mu_{34} & -\mu_{34} & 0 \\
0 & 0 & -\mu_{34} & \mu_{34}+\mu_{45} & -\mu_{45} \\
\mu_{51} & 0 & 0 & -\mu_{45} & \mu_{45}+\mu_{51}
\end{array}\right) .
$$

В случае если все коэффициенты $\mu_{i, j}>0$, то связь носит чисто диссипативный характер и привносит в систему дополнительное затухание, в случае если $\mu_{i, j}<0$, то связь активная, т.е. привносит в систему дополнительную энергию. Одной из особенностей нашей модели (и ее принципиальное отличие от [2]) - это неидентичность осцилляторов по связи. Уравнения (1) записаны таким образом, что мы можем следить за динамикой системы при вариации коэффициентов связи между каждой парой осцилляторов. Данный подход наиболее предпочтителен при экспериментальном исследовании систем, а также позволяет пронаблюдать синхронизацию между отдельными элементами ансамбля.

Модель (1) рассматривается с топологией связи кольцо, т.е. влияние друг на друга в явном виде оказывают только соседние осцилляторы, что можно видеть из матрицы связи. При малых значениях коэффициентов связи в системе будет реализовываться пятичастотный квазипериодический режим. Для того чтобы пронаблюдать переход от пятичастотных колебаний к четырехчастотным или трехчастотным, необходимо варьировать параметры системы. С целью синхронизации или захвата частот каких-либо элементов ансамбля необходимо варьировать частотную расстройку и связь. С увеличением связи в какой-то момент наступит синхронизация. Причем в случае захвата обычно перестройка режима происходит в результате седло-узловой бифуркации. Мы зафиксируем частотную расстройку в соответствии с [2], данные параметры подобраны для возможности реализации сценария Ландау-Хопфа: распределение частот осцилляторов эквидистантное. При этом коэффициенты связи между всеми осцилляторами мы не будем делать идентичными. Зафиксируем три из пяти коэффициентов связи, а коэффициенты между тремя соседними осцилляторами будем варьировать. В качестве исследуемого осциллятора мы выберем первый, и варьировать связь будем между первым и пятым осцилляторами (коэффициент $\mu_{51}$ ) и первым и вторым осцилляторами (коэффициент $\mu_{12}$ ).

На рис. 2 представлена карта показателей Ляпунова для диссипативной и активной связей. Мы целенаправленно взяли диапазон коэффициентов связи как положительный, так и отрицательный, чтобы видеть как диссипативную, так и активную связи. Соответственно фрагмент I на карте соответствует чисто диссипативной связи, фрагмент III - чисто активной связи, II и IV - 
Соответствие реализующегося в системе режима и сигнатуры спектра показателей Ляпунова, обозначение режима цветом на карте показателей Ляпунова

\begin{tabular}{c|c|c|c}
\hline Режим & Сигнатура & Спектр показателей Ляпунова & Цвет \\
\hline тор-5 & $T_{5}$ & $\Lambda_{1}=\Lambda_{2}=\Lambda_{3}=\Lambda_{4}=\Lambda_{5}=0, \Lambda_{10}<\Lambda_{9}<\Lambda_{8}<\Lambda_{7}<\Lambda_{6}<0$ & $\square-$ темно-серый \\
тор-4 & $T_{4}$ & $\Lambda_{1}=\Lambda_{2}=\Lambda_{3}=\Lambda_{4}=0, \Lambda_{10}<\Lambda_{9}<\Lambda_{8}<\Lambda_{7}<\Lambda_{6}<\Lambda_{5}<0$ & $\mathbf{\square}-$ черный \\
тор-4 & $T_{42}$ & $\Lambda_{1}=\Lambda_{2}=\Lambda_{3}=\Lambda_{4}=0, \Lambda_{10}<\Lambda_{9}<\Lambda_{8}<\Lambda_{7}<\Lambda_{6}=\Lambda_{5}<0$ & $\square-6$ елый \\
тор-3 & $T_{3}$ & $\Lambda_{1}=\Lambda_{2}=\Lambda_{3}=0, \Lambda_{10}<\Lambda_{9}<\Lambda_{8}<\Lambda_{7}<\Lambda_{6}<\Lambda_{5}<\Lambda_{4}<0$ & $\square-$ светло-серый
\end{tabular}

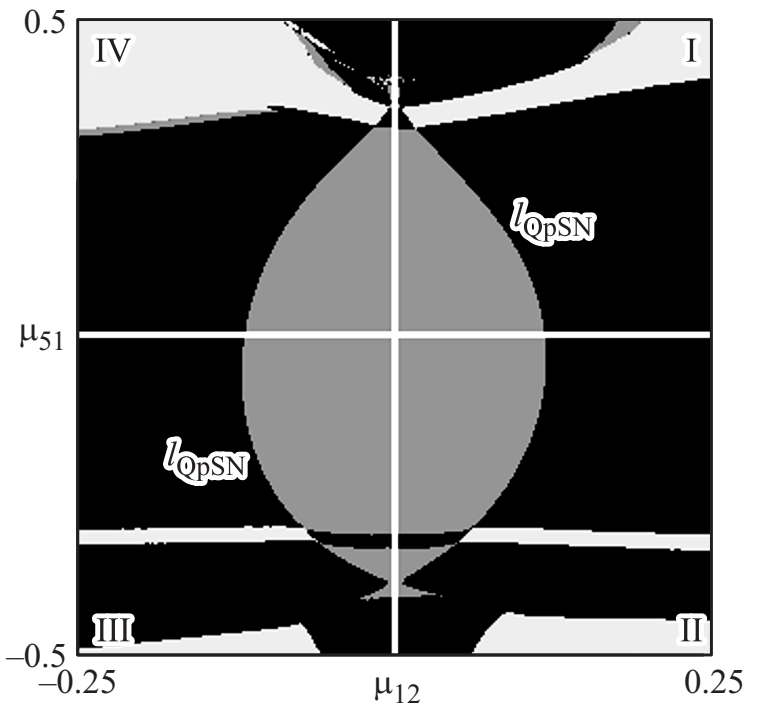

Рис. 2. Карта показателей Ляпунова для системы (1) с диссипативной и активной связью, матрица связи (3), $\mu_{23}=\mu_{34}=\mu_{45}=0.01, \lambda=0.5, \Delta=1$.

смешанные типы связи. Соответствие цветовой палитры со значениями показателей полного спектра Ляпунова и реализующимся режимом представлено в таблице.

Как видно из рисунка, в системе при малой связи реализуется пятичастотный тор $\left(T_{5}\right.$, темно-серый цвет). При увеличении параметров связи происходит синхронизация колебаний - захват частот соседних осцилляторов. При увеличении коэффициента $\mu_{12}$ синхронизуются первый и второй осцилляторы, при увеличении $\mu_{51}-$ первый и пятый. Стоит отметить, что порог синхронизации четырехчастотных торов между первым и вторым осцилляторами и между первым и пятым осцилляторами различны. Для первой пары осцилляторов $\mu_{s y n} \approx 0.12$, а для второй: $\mu_{s y n} \approx 0.41$. Данная разница связана с различием собственных частот осцилляторов. Наш ансамбль имеет эквидистантный спектр собственных частот, таким образом, частотная расстройка между первым и вторым осцилляторами составляет $25 \%$ от базовой частоты, а между первым и пятым разница в 2 раза. Следовательно, для синхронизации более разных по частоте осцилляторов требуется большая сила связи. Данные перестройки происходят в результате седло-узловой квазипериодической бифуркации. На рисунке обозначены следующие линии бифуркаций: $l_{Q p S N}-$ бифуркация седло-узел. При дальнейшем увеличении связи происходит синхронизация всех трех осцилляторов и реализуется трехчастотный квазипериодический режим.

Картина режимов для активной связи носит аналогичную структуру. Все перестроения для активной связи происходят в результате седло-узловой бифуркации. Также пороги синхронизации пар осцилляторов отличаются для каждой пары и немного превышают по значению случай диссипативной связи.

В работе [2] продемонстрирован сценарий ЛандауХопфа: последовательное возникновение частот в спектре через квазипериодические бифуркации Хопфа. Одним из принципиальных факторов для реализации такого сценария является различный уровень диссипации в элементах ансамбля (для одиночных подсистем - это параметр $\lambda$, отвечающий за возбуждение автоколебаний). В ансамбль диссипация привносится не только параметром $\lambda$, но и самой связью. Оставляя параметры $\lambda$ фиксированными и идентичными для всех осцилляторов, можно варьировать коэффициенты связи и таким образом изменять уровень диссипации в системе.

Как мы уже видели, для случаев диссипативной и активной связей квазипериодической бифуркации Хопфа при переходе от 5-тора к 4-тору не наблюдается. Попробуем немного изменить количество диссипации в системе с помощью изменения знака одного из слагаемых в матрице связи. Изменим знак у одного из слагаемых связи в первом осцилляторе. В нашем случае мы изменим знак диссипации, идущей от второго осциллятор в первый. При этом знаки диссипации, поступающей от того же осциллятора в другой, оставим прежними. Матрица связи в этом случае примет вид

$$
\mu_{i j}^{2}=\left(\begin{array}{ccccc}
\mu_{51}-\mu_{12} & +\mu_{12} & 0 & 0 & -\mu_{51} \\
-\mu_{12} & \mu_{12}+\mu_{23} & -\mu_{23} & 0 & 0 \\
0 & -\mu_{23} & \mu_{23}+\mu_{34} & -\mu_{34} & 0 \\
0 & 0 & -\mu_{34} & \mu_{34}+\mu_{45} & -\mu_{45} \\
-\mu_{51} & 0 & 0 & -\mu_{45} & \mu_{45}+\mu_{51}
\end{array}\right)
$$

На рис. 3 представлена карта показателей Ляпунова для системы (1) со связью, описываемой матрицей (3). Как видно из рисунка, при малой связи в системе так же наблюдается пятичастотный тор. При увеличении связи происходит синхронизация между парой осцилляторов, также как и для первого случая, и в системе реализуется четырех частотный квазипериодический режим. Черный 


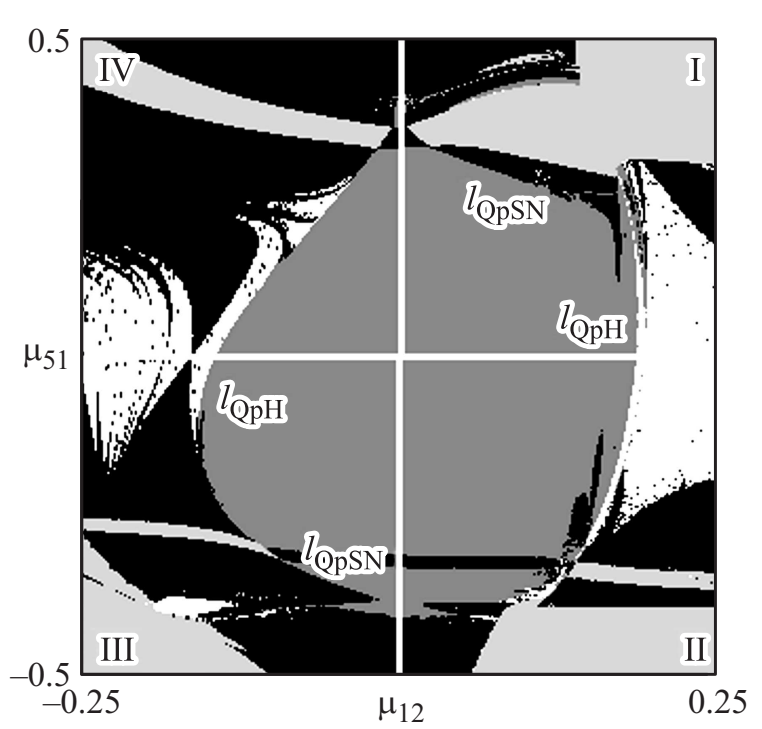

Рис. 3. Карта показателей Ляпунова для системы (1) с диссипативной и активной связью с единичным инверсным знаком, матрица связи (4), $\mu_{23}=\mu_{34}=\mu_{45}=0.01, \lambda=0.5$, $\Delta=1$.

и белый цвета обозначают 4-тор, если переход от темносерой области (5-тор) происходит в черную (4-тор) при вариации параметров, то это соответствует квазипериодической бифуркации седло-узел, если переход в белую область, то это соответствует квазипериодической бифуркации Хопфа $\left(l_{Q p H}-\right.$ линии бифуркации Хопфа). Как видно из рисунка, основные режимы, реализующиеся в системе при инверсии знака связи, остались теми же: 5-тор, 4-тор, 3-тор. Однако появилась квазипериодическая бифуркация Хопфа при переходе от 5-тора к 4-тору. Квазипериодическая бифуркация Хопфа происходит при увеличении связи того осциллятора, знак связи с которым был инвертирован. Это означает, что синхронизация с осцилляторами, которые связаны обычной связью, происходит таким же образом, что и для обычной связи через квазипериодическую бифуркацию седло-узел. При этом для единственного осциллятора в ансамбле, для которого был изменен знак связи, переход от пятичастотного квазипериодического режима к четырехчастотному происходит через квазипериодическую бифуркацию Хопфа.

Таким образом, в работе представлено исследование динамики пяти связанных в кольцо осцилляторов ван дер Поля. Рассмотрены особенности перехода от пятичастотного квазипериодического режима к четырехчастотному. Обнаружена специфическая топология связи осцилляторов, позволяющая реализовать квазипериодическую бифуркацию Хопфа в системе с идентичными внутренними параметрами за счет изменения типа связи.

Работа выполнена при финансовой поддержке РФФИ (грантов № 14-02-00085, № 16-02-00135) и гранта Президента РФ (проект № МК-661.2017.8).

\section{Список литературы}

[1] Кузнецов А.П., Сатаев И.Р., Станкевич Н.В., Тюрюкина Л.В. Физика квазипериодических колебаний. Саратов: Издательский центр „Наука“, 2013.

[2] Kuznetsov A.P., Kuznetsov S.P., Sataev I.R., Turukina L.V. // Phys. Lett. A. 2013. Vol. 377. P. 3291-3295.

[3] Sekikawa M., Inaba N., Kamiyama K., Aihara K. // Chaos. 2014. Vol. 24. N 1. P. 013137.

[4] Rosenblum M., Pikovsky A. // Phys. Rev. E. 2015. Vol. 92. P. 012919.

[5] Кондрашов А.В., Устинов А.Б., Калиникос Б.А. // Письма в ЖТФ. 2016. Т. 42. Вып. 4. С. 82-89.

[6] Anchikov D.A., Shakirov A.P., Krents A.A., Molevich N.E., Pakhomov A.V. // Physics of Wave Phenomena. 2016. Vol. 24. N 2. P. 1-6.

[7] Broer H., Simó C., Vitolo R. // Regul. Chaotic Dyn. 2011. Vol. 16. P. 154-184. 